\title{
The nomogram establishment of thermal conductivity and strength of insulating concrete for high temperature mine
}

\author{
Hongwei Wang ${ }^{1}$ and Shuguang Zhang, ${ }^{2, *}$ \\ ${ }^{1}$ Institute of science and technology, Taiyuan University of Technology, 030024 Taiyuan,China \\ ${ }^{2}$ School of civil and architectural engineering, Guilin University of Technology, 541004 Guilin,China
}

\begin{abstract}
The insulating concrete can be effectively reduced heat exchange between surrounding rock and ventilation air, which is of positive significance to mine cooling and energy conservation. The law of the mixing amount of insulation material on thermal conductivity and strength was discovered through testing 75 specimens of insulating concrete used for mine. Based on new effective medium theory, calculation model of coefficient of heat conductivity is deduced, and its parameters are acquired with the surface regression analysis method, then the mixing threshold of insulation material is determined. The resulting experimental data and calculational results make up data sample, and then the nomogram of thermal conductivity and strength of insulating concrete is plotted. The nomogram curve can be divided into regions with the main intensity and heat insulation as the auxiliary, the mutual influence region and the main intensity and heat insulation as the auxiliary. The constructed nomogram would not only provide the basis for determining porosity of compound concrete, but also determine thermal conductivity and strength according to porosity. It can simplify calculation step for the engineering and technical staffs, and help to guide design of insulating concrete.
\end{abstract}

\section{Introduction}

Mine high temperature is an irreversible thermodynamic process under the action of complex engineering geological environment. High temperature damage not only causes deterioration of working environment, but also is a potential threat to safe mining[1]. The heat insulation structure adopted in high temperature mine can effectively reduce the heat dissipation from surrounding rock to air flow, and save the amount of mine cooling to achieve energy saving and emission reduction[2].

The thermal conductivity of concrete is affected by many factors such as age, water - cement (W/C) ratio, types of admixtures, aggregate volume fraction, fine aggregate faction, temperature, and humidity condition of specimen[3]. It has been confirmed that thermal conductivity and compressive strength of concrete incorporation with mineral admixtures[4-6].Combined with the basic theories of classical fluid mechanics and heat transfer, the rock-soil mass is simplified as a continuous medium with solid, liquid and gas threephase composition, uniform distribution and isotropy, and the mathematical model of heat transfer are established[7-10]. The thermal network concept and Fourier's law are used to develop a mathematical model to calculate the effective thermal conductivity of reinforced concrete with different numbers of round rebars oriented either normal or parallel to the heattransfer direction, and different volume fractions of steel[11,12]. Based on the diversity of composite concrete, the artificial neural networks approaches has also been used to estimate thermal conductivity of concrete[13,14]. Then, factors affecting thermal conductivity are studied such as material structure, damage evolution, water-cement ratio, aggregate, porosity [15-19]. In recent years, research on correlation between strength and thermal conductivity of composite concrete is growing $[20,21]$.

It can be seen that the calculation of thermal conductivity parameters involves many factors and the calculation process is complex, so it is particularly necessary to establish a simple and feasible determination method to meet the needs of the engineering practice. The supporting concrete used in high-temperature mine roadway is different from ordinary insulating concrete, which requires both the strength to meet the needs of the project and the lowest thermal conductivity.

This article uses the method of combining the experimental research and model calculation, the change rule of coefficient of thermal conductivity and strength of the insulating concrete are studied under the condition of different heat insulation material and mixing amount. Furthermore, the thermal conductivity of thermal insulating concrete in high-temperature mine was constructed, so as to simplify the calculation process of engineering technicians. Research results can provide basis for engineering application of heat insulation concrete support technology in high temperature mine.

\footnotetext{
* Corresponding author: fx5152@126.com
} 


\section{Preparation of insulating concrete specimen}

\subsection{Material selection}

Insulating concrete is a porous medium composed of solid skeleton, insulating material, void and water. Compared with ordinary porous materials, the purpose of adding ceramic particles, vitrified micro bubbles and other thermal insulation materials is to change the original pore structure of concrete, and make the thermal conductivity of the solid skeleton tend to be heterogeneous, so as to meet the technical requirements and strength requirements of mine shotcrete, and to reduce its thermal conductivity as far as possible.

\subsubsection{Glazed hollow bead}

Glazed hollow bead(GHB) is irregular spherical particles with hollow inside and smooth surface after high temperature melting of volcanic ores. Its internal structure is stable and has excellent physical and chemical properties, and it is a kind of inorganic heat insulation material with light weight and environmental protection. GHB have some open and unclosed pores, which make it easy to absorb water and reduce the strength of concrete. Because styrene-acrylic emulsion has the advantages of fast drying, water resistance, alkali resistance and washing resistance, a waterproof and alkali resistant transparent film on its surface is formed (Fig.1) after uniformly mixed with the vitrified micro bubbles. Modified GHB can effectively seal the void and reduce water adsorption.

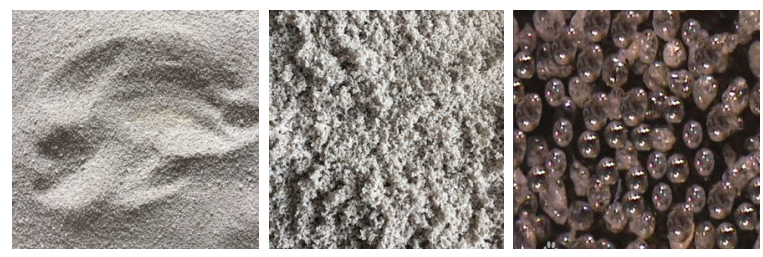

Fig. 1. Comparison diagram of before modification and modified GHB.

\subsubsection{Shale ceramsite}

Shale ceramsite is a granular ceramsite made of shale, clay rock, fly ash and gangue through crushing, screening, high temperature and roasting. The surface of the ceramsite is rough and hard, with good tightness, more internal voids, greater porosity and other characteristics. The size of the ceramsite selected in the test was more than $5 \mathrm{~mm}$, the thermal conductivity was $0.25-0.45 \mathrm{w} /(\mathrm{m} \cdot \mathrm{K})$, the porosity was about $60 \%$, the bulk density was $0.36-0.8 \mathrm{~g} / \mathrm{cm}^{3}$, and the cylinder compressive strength was $5.1 \mathrm{MPa}$.

\subsubsection{Other materials}

There are many kinds of cement to choose from. The ordinary 42.5 Portland cement is selected, which has rapid setting and hardening speed, good antifreeze performance, high strength and low price.Gravel with medium diameter in the range of $5-16 \mathrm{~mm}$ is adopted, with a soil content of $0.5 \%$ and a packing density of $1500 \mathrm{~kg} / \mathrm{m}^{3}$. Fine aggregate is medium sand with fineness modulus 2.5 , mud content $3.0 \%$, packing density of 1530 $\mathrm{kg} / \mathrm{m}^{3}$. The selected water is tap water, and the amount of concrete accelerating agent is about $4 \%$ of cement.

\subsection{Specimen preparation}

The proportion of specimen is designed according to the technical specification of shotcrete support engineering (Table1). The overall scheme was as follows: watercement ratio(W/C) was set as 0.46 0.50; sand ratio was set at $50 \%$; GHB are added as fine aggregate, and their contents are respectively $3 \%, 6 \%, 9 \%, 12 \%$ and $15 \%$ of the amount of substitute fine aggregate; shale ceramsite was added as coarse aggregate, and its content was $5 \%$, $10 \%, 15 \%, 20 \%$ and $25 \%$ of the dosage of substitute coarse aggregate respectively; the slump was controlled at $(10 \pm 2) \mathrm{cm}$.

Table 1. Standard ration of thermal insulation concrete.

\begin{tabular}{|c|c|c|c|c|c|}
\hline Group & $\mathrm{W} / \mathrm{C}$ & $\begin{array}{c}\text { Cement } \\
/ \mathrm{kg} \cdot \mathrm{m}^{-3}\end{array}$ & $\begin{array}{c}\text { Fine } \\
\text { aggregate } \\
/ \mathrm{kg} \cdot \mathrm{m}^{-3}\end{array}$ & $\begin{array}{c}\text { Coarse } \\
\text { aggregate } \\
/ \mathrm{kg} \cdot \mathrm{m}^{-3}\end{array}$ & $\begin{array}{c}\text { Water } \\
/ \mathrm{kg} \cdot \mathrm{m}^{-3}\end{array}$ \\
\hline$a$ & 0.50 & 542 & 801 & 801 & 271 \\
\hline$b$ & 0.48 & 500 & 730 & 730 & 240 \\
\hline$c$ & 0.46 & 478 & 688 & 688 & 220 \\
\hline
\end{tabular}

The method of preparation is a combination of concrete mixer and vibrating table. In order to reduce the breakage rate of GHB in the mixing process, GHB were added in the middle and later stages of the whole mixing process. First, cement, gravel, sand and ceramsite were put into the concrete mixer for 30 seconds, and appropriate amount of water was added for stirring $1 \mathrm{~min}$. Then, GHB were uniformly poured into the concrete mixer for stirring $1 \mathrm{~min}$. Finally, accelerator was added at the ratio of $4 \%$ cement content for stirring 30 seconds.

After pouring the mixture into the mold and smoothing, the plastic film is immediately covered its surface to prevent the rapid evaporation of water from affecting the compressive strength of the specimen. The specimens are demoulded and numbered after 24 hours in $(20 \pm 5)^{\circ} \mathrm{C}$, then the standard specimens are made after curing $28 \mathrm{~d}$ in curing box with temperature $(20 \pm 2)^{\circ} \mathrm{C}$ and relative humidity $95 \%$.

\section{Performance test of the specimen}

For the ratio of three groups in Table1, 75 sets of samples were made and thermal conductivity and strength were tested, the test results of strength and thermal conductivity are shown in Table 2 . 
Table 2. The test results of strength and thermal conductivity of samples with different proportions

\begin{tabular}{|c|c|c|c|c|c|c|c|}
\hline \multirow{3}{*}{$\begin{array}{c}\text { GHB content } \\
/ \%\end{array}$} & \multirow{3}{*}{$\begin{array}{l}\text { Ceramsite } \\
\text { content } / \%\end{array}$} & \multicolumn{6}{|c|}{ Water cement ratio } \\
\hline & & \multicolumn{2}{|c|}{0.50} & \multicolumn{2}{|c|}{0.48} & \multicolumn{2}{|c|}{0.46} \\
\hline & & \begin{tabular}{|c|} 
Thermal \\
conductivity \\
$/ \mathrm{W} /(\mathrm{m} \cdot \mathrm{K})$
\end{tabular} & $\begin{array}{l}\text { Compressive } \\
\text { strength/MPa }\end{array}$ & $\begin{array}{c}\text { Thermal } \\
\text { Conductivity/ } \\
\mathrm{W} /(\mathrm{m} \cdot \mathrm{K})\end{array}$ & $\begin{array}{l}\text { Compressive } \\
\text { strength/MPa }\end{array}$ & $\begin{array}{c}\text { Thermal } \\
\text { Conductivity/ } \\
\mathrm{W} /(\mathrm{m} \cdot \mathrm{K})\end{array}$ & $\begin{array}{l}\text { Compressive } \\
\text { strength/MPa }\end{array}$ \\
\hline \multirow{5}{*}{3} & 5 & 1.09 & 32.29 & 1.09 & 32.31 & 1.09 & 32.32 \\
\hline & 10 & 0.91 & 30.84 & 0.91 & 30.87 & 0.91 & 30.90 \\
\hline & 15 & 0.75 & 29.46 & 0.75 & 29.50 & 0.75 & 29.54 \\
\hline & 20 & 0.62 & 28.15 & 0.62 & 28.21 & 0.62 & 28.26 \\
\hline & 25 & 0.50 & 26.92 & 0.50 & 26.98 & 0.51 & 27.05 \\
\hline \multirow{5}{*}{6} & 5 & 0.41 & 25.76 & 0.41 & 25.84 & 0.42 & 25.91 \\
\hline & 10 & 0.35 & 24.68 & 0.35 & 24.76 & 0.35 & 24.85 \\
\hline & 15 & 0.30 & 23.67 & 0.30 & 23.76 & 0.30 & 23.85 \\
\hline & 20 & 0.28 & 22.73 & 0.28 & 22.83 & 0.28 & 22.93 \\
\hline & 25 & 0.28 & 21.87 & 0.28 & 21.98 & 0.28 & 22.09 \\
\hline \multirow{5}{*}{9} & 5 & 0.30 & 21.08 & 0.30 & 21.20 & 0.30 & 21.31 \\
\hline & 10 & 1.09 & 31.77 & 1.09 & 31.96 & 1.10 & 32.15 \\
\hline & 15 & 0.91 & 30.33 & 0.91 & 30.53 & 0.92 & 30.72 \\
\hline & 20 & 0.75 & 28.97 & 0.76 & 29.17 & 0.76 & 29.37 \\
\hline & 25 & 0.62 & 27.68 & 0.62 & 27.89 & 0.62 & 28.10 \\
\hline \multirow{5}{*}{12} & 5 & 0.50 & 26.47 & 0.51 & 26.68 & 0.51 & 26.89 \\
\hline & 10 & 0.41 & 25.33 & 0.42 & 25.54 & 0.42 & 25.76 \\
\hline & 15 & 0.34 & 24.26 & 0.35 & 24.48 & 0.35 & 24.70 \\
\hline & 20 & 0.30 & 23.27 & 0.30 & 23.49 & 0.31 & 23.71 \\
\hline & 25 & 0.28 & 22.35 & 0.28 & 22.58 & 0.28 & 22.80 \\
\hline \multirow{5}{*}{15} & 5 & 0.28 & 21.51 & 0.28 & 21.74 & 0.28 & 21.96 \\
\hline & 10 & 0.30 & 20.74 & 0.30 & 20.97 & 0.30 & 21.20 \\
\hline & 15 & 1.09 & 31.24 & 1.10 & 31.60 & 1.11 & 31.96 \\
\hline & 20 & 0.91 & 29.82 & 0.92 & 30.18 & 0.93 & 30.54 \\
\hline & 25 & 0.75 & 28.48 & 0.76 & 28.84 & 0.77 & 29.19 \\
\hline
\end{tabular}


Test results show that GHB and ceramsite have obvious influence on the thermal conductivity and strength of concrete. When the proportion of ceramsite content is $15 \%-25 \%$, the influence on the thermal conductivity is more obvious; on the contrary, the influence on the strength is relatively poor, which is an ideal dosage threshold.As the content of GHB increases, the thermal conductivity and strength decrease to different degrees. When the content of GHB reaches $15 \%$, the compressive strength drops below $20 \mathrm{MPa}$. Therefore, it is suggested that the content of GHB should be controlled at $6 \%-12 \%$.

\section{Heat transfer model and parameter determination}

\subsection{Heat transfer model}

According to the basic principle of the new effective medium theory (Fig.2), the phases comprising porous materials are regarded as small spheres dispersed into a hypothetical homogeneous medium with a thermal conductivity of $k_{m}$. If the material has an $i$ phase, the phase $i$ is regarded as $\mathrm{n}$ small spheres with radius $R_{i}$ and thermal conductivity $k_{i}$, which are contained in homogeneous medium with thermal conductivity $k_{m}$.

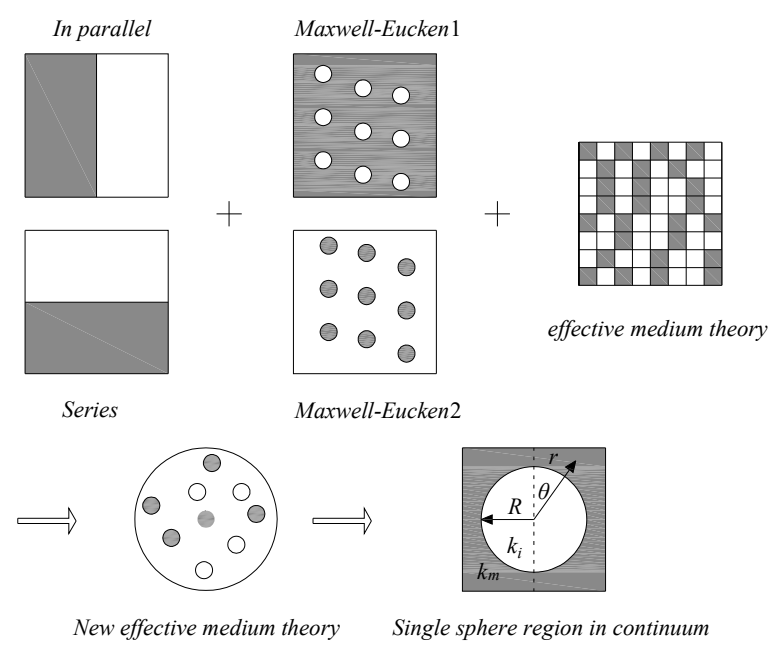

Fig. 2. Schematic illustration of new effective medium theory

The temperature distribution of a single sphere in a uniform medium under steady state condition satisfies Laplace equation:

$$
\frac{1}{r^{2}} \frac{\partial}{\partial_{r}}\left(r^{2} \frac{\partial T}{\partial_{r}}\right)+\frac{1}{r^{2} \sin \theta} \frac{\partial}{\partial \theta}\left(\sin \theta \frac{\partial T}{\partial \theta}\right)=0
$$

The boundary condition is: when $r=0, T_{i} \neq \infty$; when $r=R_{i}, k_{i} \frac{\partial T_{m}}{\partial r}=k_{m} \frac{\partial T_{m}}{\partial r}$ and $T_{m}=b r \cos \theta$.

There, $r$ and $\theta$ is the polar radius and the polar Angle in polar coordinates, and $T$ is temperature, and constant $b$ is the magnitude of the temperature gradient in the continuous medium, and the subscripts $i$ and $m$ represent the phase $i$ and the assumed homogeneous medium, respectively.
The above equation is analyzed and solved in literature [22], and the results are as follows:

$$
\sum v_{i}\left(\frac{k_{i}-k_{e}}{k_{i}+2 k_{m}}\right)=0
$$

Heat insulation concrete is regarded as a combination of skeleton and porosity. Equation (2) is converted into:

$$
(1-\varepsilon) \frac{k_{s}-k_{e}}{k_{s}+2 k_{m}}+\varepsilon \frac{k_{a}-k_{e}}{k_{a}+2 k_{m}}=0
$$

There, $k$ is the thermal conductivity coefficient; $e$ is porosity; the subscripts $e, a$ and $s$ respectively represents the effective medium, air and solid.

Since the continuous phase thermal conductivity of concrete adopted in this paper is greater than that of the dispersed phase, So $k_{m}=k_{s}$, then an effective thermal conductivity model suitable for insulating concrete is obtained:

$$
k_{e}=k_{s} \frac{2 k_{s}+k_{a}-2\left(k_{s}-k_{a}\right) \varepsilon}{2 k_{s}+k_{a}+\left(k_{s}-k_{a}\right) \varepsilon}
$$

There, $k_{s}$ is the thermal conductivity of the continuous phase in the porous structure, that is, the thermal conductivity of the solid phase. According to the definition of the thermal conductivity, the product of the volume fraction of each doped material in the porous structure and their thermal conductivity is the thermal conductivity of the solid phase.

According to the experimental results in this paper, the heat-insulating concrete can be simplified into a mixed porous structure composed of glass bubbles, ceramsite and ordinary concrete, namely $k_{s}$ :

$$
k_{s}=\frac{b m_{1}}{V \rho_{b}} k_{b}+\frac{c m_{2}}{V \rho_{c}} k_{c}+\left(1-\frac{b m_{1} \cdot \rho_{b}^{-1}+c m_{2} \cdot \rho_{c}^{-1}}{V}\right) k_{e}
$$

There, $V=\sum m_{i} / \rho_{i}$ is the sum of the volumes of the insulating concrete admixture $i ; m_{1}$ and $m_{2}$ are fine aggregate and coarse aggregate contents respectively; $P_{b}$ and $\rho_{c}$ are the density of vitrified microbeads and ceramsite respectively; $k_{e}$ is the thermal conductivity of ordinary concrete; $b$ and $c$ are the mixing amount of glass bubbles and ceramsite respectively. Thermal conductivity of GHB $k_{b}=0.036 \mathrm{~W} /(\mathrm{m} \cdot \mathrm{K})$, that of ceramsite $k_{c}=0.35 \mathrm{~W} /(\mathrm{m} \cdot \mathrm{K})$, that of air $k_{a}=0.0267$ $\mathrm{W} /(\mathrm{m} \cdot \mathrm{K})$, that of concrete $k_{e}=1.28 \mathrm{~W} /(\mathrm{m} \cdot \mathrm{K})$. The density of GHB was $90 \mathrm{~kg} / \mathrm{m}^{3}$, that of ceramsite was $780 \mathrm{~kg} / \mathrm{m}^{3}$, that of sand was $1530 \mathrm{~kg} / \mathrm{m}^{3}$, that of crushed stone was $1,500 \mathrm{~kg} / \mathrm{m}^{3}$, and that of cement was $3,000 \mathrm{~kg} / \mathrm{m}^{3}$.

\subsection{Parameter determination}

In the effective thermal conductivity model of insulated concrete, the thermal conductivity ks and porosity e of solid phase are variables related to the proportion of glass bubbles and ceramsite.

Matlab was used to fit the relationship between the solid thermal conductivity $k_{s}$ and the proportion $b$ of GHB and ceramsite $c$ respectively (Fig.3), the relationship between porosity $\varepsilon$ and the proportion of GHB and ceramsite respectively (Fig.4), and the relationship between compressive strength and the proportion of GHB and ceramsite respectively (Fig.5). 
The surface equation of the relation between the thermal conductivity $k_{s}$ of solid phase and the proportion $b$ and $c$ of glass bubbles and ceramsite contents is as follows:

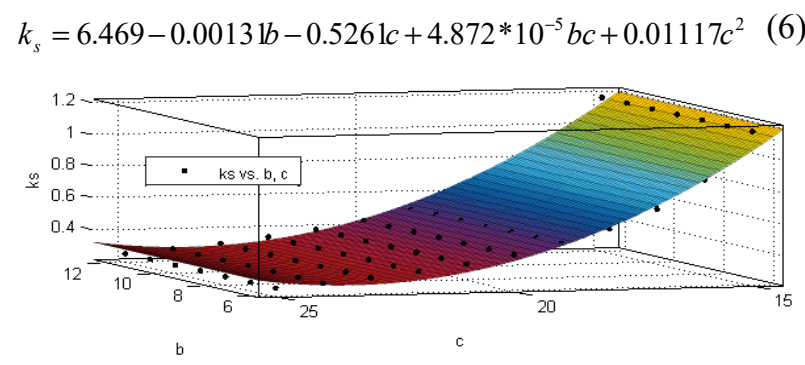

Fig.3. The relationship between the thermal conductivity of solid phase ks and the content of heat insulation material

The surface equation of the relationship between porosity and proportion $b$ and $c$ of vitrified beads and ceramsite content is as follows:

$$
e=7.931+0.1304 b+0.1192 c
$$

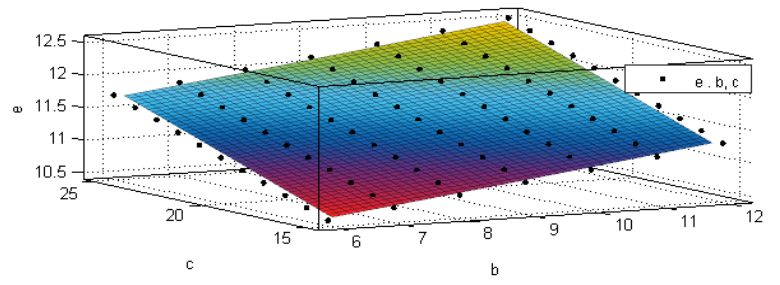

Fig.4. The relationship between the porosity $e$ and the content of the heat insulation material

Put equations (6) and (7) after regression into equation (4), the thermal conductivity of concrete with different amounts of heat insulation materials can be calculated.

The relationship between compressive strength and the specific gravity of glass bubbles and ceramsite content is:

$$
P=67.84-0.8075 b-2.715 c+0.01877 b c+0.03703 b^{2}
$$

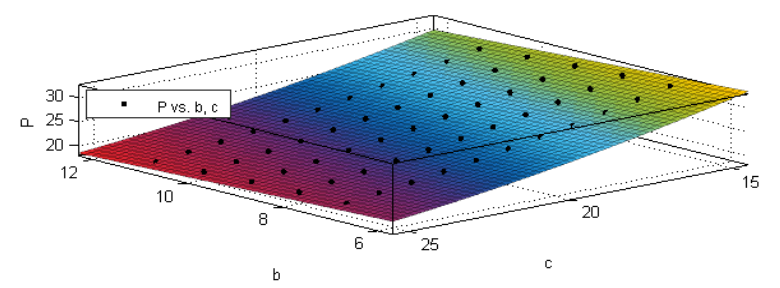

Fig.5. The relationship between compressive strength and the content of heat insulation material

\section{Nomogram construction}

\subsection{The determination of the coordinate system of nomogram}

The function of nomogram is to provide a method to quickly find the thermal conductivity and compressive strength of insulating concrete by image, So determine the coefficient of thermal conductivity and calculate the two ordinates of the norm diagram as the coefficient of thermal conductivity and compressive strength.GHB and ceramsite are used as thermal insulation materials to add thermal insulation concrete. In fact, the effect of adding thermal insulation concrete is to artificially change its pore structure, so the $\mathrm{x}$-coordinate is set as the porosity of thermal insulation concrete.

\subsection{The drawing of nomogram}

According to the experimental determination that the mixing amount of glass bubbles is $6 \%-12 \%$ and the proportion of ceramsite mixing amount is $15 \%-25 \%$, all the test data points meeting the strength requirements are first drawn in the nomogram coordinate system, and then the thermal conductivity and compressive strength of insulating concrete with other contents are calculated step by step. The experimental results and calculation results were drawn to form a scatter diagram in the coordinate system of nomogram. On this basis, the threshold values of thermal conductivity and compressive strength and the data lines of the mixing amounts of glass bubbles were drawn. The drawing process of nomogram was shown in Fig.6.

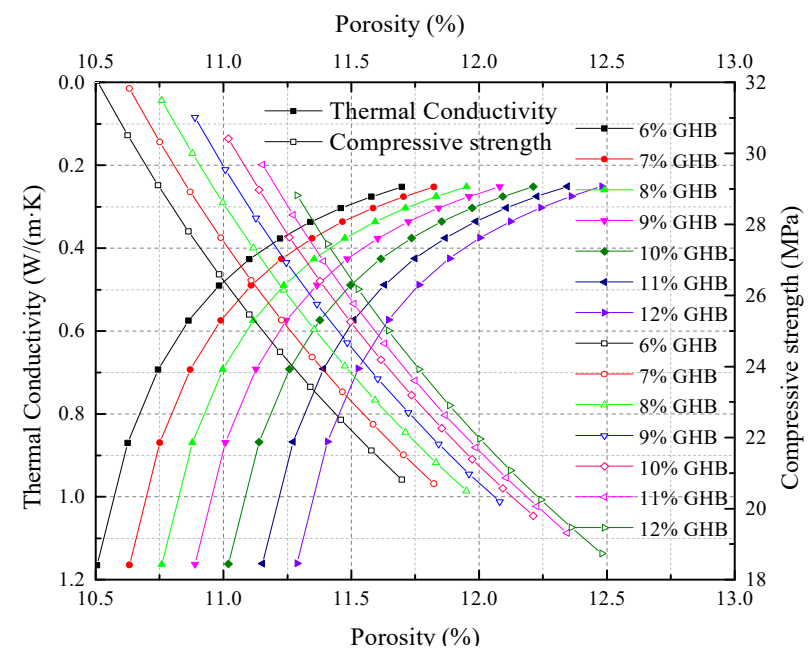

Fig.6. The nomogram of thermal conductivity and strength of insulating concrete

The results of nomogram show that the strength of insulating concrete decreases monotonously with the increase of porosity, and the change rate of thermal conductivity is greater when the content of ceramsite is $15 \%-18 \%$ than when the content of ceramsite is $18 \%$ $25 \%$. When the porosity of insulating concrete is lower than $11 \%$, the compressive strength is greater than $26 \mathrm{MPa}$, and the thermal conductivity is not less than $0.5 \mathrm{w} /(\mathrm{m} \cdot \mathrm{K})$; The compressive strength and thermal conductivity in this area are both high, which is suitable for the design of reinforced concrete. When the porosity is $11.0 \%-11.6 \%$, the thermal conductivity and the strength influence each other. Although the porosity is the same in this region, the results of different content of heat insulation materials are quite different. In particular, the increase in the content of GHB will lead to a significant decrease in the strength. When the porosity is 
greater than $11.6 \%$, the compressive strength and thermal conductivity both show a significant reduction, suitable for the design of heat insulation primarily for the auxiliary strength of concrete; In this high porosity area, since the maximum mixing amount of GHB is controlled at $12 \%$ according to the strength requirements, the contribution of porosity mainly comes from ceramsite. From the reduction of thermal conductivity, the closed pore structure of ceramsite has a more significant effect on thermal insulation.

\subsection{The use of nomogram}

The nomogram can be used in two ways:

(1)If the amount of heat insulation material is known, the porosity of heat insulation concrete can be converted, so that the thermal conductivity and compressive strength range of heat insulation concrete can be directly obtained according to the porosity.

(2)After the strength of insulating concrete is determined according to the project needs, the thermal conductivity of insulating concrete is selected as required, and the porosity can be obtained from the figure, so as to determine the mixing amount of glass bubbles and ceramsite.

\section{Conclusion}

Through the experimental study on the thermal conductivity and strength of insulating concrete, the heat transfer model was established and the model parameters were determined. Based on the test and calculation results, the nomogram was constructed. The main conclusions are as follows:

(1)Based on the test results and model calculation results, the influence law of heat insulation material content on thermal conductivity and strength of heat insulation concrete is obtained.According to the requirements of strength and thermal conductivity, it is suggested that the mixing threshold of glazed hollow bead in fine aggregate is $6 \%-12 \%$, and that of ceramsite in coarse aggregate is $15-25 \%$.

(2)According to the influence of the heat insulation material content on the thermal conductivity and strength of heat insulation concrete, the nomogram curve can be divided into three parts: the main strength heat insulation is auxiliary area, the mutual influence area and the main strength heat insulation is auxiliary area, so as to preliminarily determine the porosity range of prepared concrete according to the engineering needs.

(3)The nomogram of insulated concrete is constructed, which is convenient for engineers to determine the thermal conductivity and strength of insulated concrete, and helpful to guide the design of insulated concrete for mine.

\section{Acknowledgement}

This research was financially supported by the National Science Foundation of China $(51604142,51574141)$.

\section{References}

1. Z. G. Su, Z. A. Jiang, Z. Q. Sun, Earth Planet. Sci. 1, 1(2009)

2. X. F. Wang, M. Z. Gao, Q. Wang, Y. Li, Applied Mechanics \& Materials (2013)

3. K. H. Kim, S. E. Jeon, J. K. Kim, S. C. Yang, Cem. Concr. Res. CCNRAI M 33, 3(2003)

4. D. Ramazan, Build. Environ. 42, 7(2007)

5. O. Sengul, S. Azizi, F. Karaosmanoglu, M. AliTasdemir, Energy Build. 43, 2-3 (2011)

6. R. Demirboga, R. Gul, Cem. Concr. Res. CCNRAI M 33, 5 (2003)

7. B. Alazmi, K. Vafai, J. Teat Transfer, 122, 2(2000)

8. X. Y. Liu, C. Y. Zheng, X. P. Zhang. J. Eng. Thermophys., 31, 3(2010)

9. D. H. Xia, S. S. Guo, L. gRen, J. Univ. Sci. Technol. B. 34, 6(2012)

10. H. W. Wang, H. Rong, J. CHINA Coal Soc. 38, 10(2013)

11. H. G. Noh, H. C. Kang, M. H. Kim, H. S. Park, Int. J. Concr. Struct. Mater. 12, 65(2018)

12. W. P. Zhang, H.G. Min, X. L. Gu,Y. P. Xi, Y. S. Xing, Construct. Build. Mater. 98, 8-16(2015)

13. O. Gencel, F. Koksal, M. Sahin, M. Y. Durgun, H. E. Hagg Lobland, W. Brostow, Exp. Heat Transfer 26, 4(2013)

14. J. H. Lee, J. J. Lee, B. S. Cho, Int. J. Concr. Struct. Mater. 6, 3(2012)

15. S. B. Tang, C. A. Tang, Z. Z. Liang, Y. B. Zhang, L. C. Li, Heat Transfer Eng. 33, 8(2012)

16. Z. Perkowski, Bauphysik, 30, 6(2010)

17. W. Zhang, F. Tong, Y. Xing, X. Gu, J. Build. Mater. 18, 2(2015)

18. G. Pia, U. Sanna, Construct. Build. Mater. 44(2013)

19. D. F. Nurgaliev, V. M. Sizyakov, V. A. Utkov, Refract. Ind. Ceram. 55, 4(2014)

20. M. H. Niaki, A. Fereidoon, M. G. Ahangari, Compos. Struct. 191(2018)

21. K. Emsettin, D. Metin, A. Mehmet, Arab. J. Geosci. 11, 11(2018)

22. L. L. Gong, Y. H. Wang, X. D. Cheng, Int. J. Heat Mass Tran. 68(2014) 